Hepatobiliary Cancer

\title{
Clinical Profile and Treatment of Hepatocellular Carcinoma: A Single-Center Experience
}

\author{
Abu B. H. Bhatti ${ }^{1}$ Abdul A. E. Sheikh ${ }^{1} \quad$ Umair S. Mahmud ${ }^{1} \quad$ Shagufta Zeeshan $^{1} \quad$ Nusrat Y. Khan ${ }^{1}$ \\ Haseeb H. Zia ${ }^{1}$ Faisal S. Dar ${ }^{1}$ Atif Rana
}

${ }^{1}$ Department of Hepato-Pancreato-Biliary Surgery and Liver

Transplantation, Shifa International Hospital, Islamabad, Pakistan

2Department of Radiology, Shifa International Hospital, Islamabad, Pakistan
Address for correspondence Abu B. H. Bhatti, FCPS, Department of Hepato-Pancreato-Biliary Surgery and Liver Transplantation, Shifa International Hospital, Islamabad 44000, Pakistan (e-mail: abubakar.hafeez@yahoo.com).

South Asian J Cancer 2021;10:76-80.

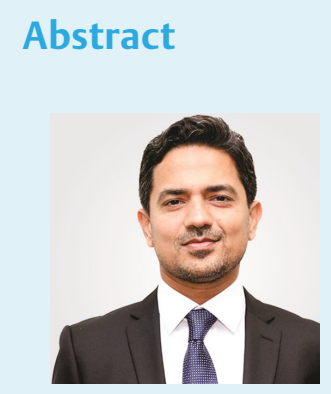

Abu Bakar H. Bhatti
Keywords

- Barcelona clinic liver cancer staging

- hepatocellular carcinoma

- liver transplantation

- transarterial chemoembolization
Background Very few centers in Pakistan have all established treatments for hepatocellular carcinoma (HCC) available under one roof. With a dedicated hepato-pancreato-biliary surgery and liver transplant unit, we have gathered one of the largest data on HCC in our population.

Aims The objective of the current study was to assess the clinical spectrum of HCC in Pakistani patients.

Settings and Design This retrospective review of patients diagnosed with HCC was conducted between 2011 and 2016.

Materials and Methods Patients were allocated to treatment groups based on the Barcelona clinic liver cancer (BCLC) staging algorithm and our local guidelines. The treatment options were grouped as curative (radiofrequency ablation [RFA], percutaneous ethanol injection [PEI], liver resection, and liver transplantation), palliative (transarterial chemoembolization [TACE]/sorafenib), and the best supportive care (BSC). Statistical Analysis Kaplan-Meier curves were used for the statistical analysis.

Results The mean age was $57.9 \pm 10.1$ years (range: $18-90$ years). The male-to-female ratio was (1,099/391) 2.8:1. Hepatitis $B$ and hepatitis $C$ were the most common underlying etiological factor in 1,350 of 1,490 (90.6\%) patients. Macrovascular invasion (MVI) was seen in 492 of 1,490 (33\%) patients. Out of the total, 191 (12.8\%) additional patients were offered potentially curative treatments when compared with BCLC recommendations. The actuarial 5-year overall survival for patients who underwent liver transplant, RFA/PEI, TACE, sorafenib, and BSC was 87, 64, 18, 5, and 0\%, respectively. Alpha fetoprotein cut-off of $400 \mathrm{ng} / \mathrm{mL}$ had a significant impact on survival irrespective of treatment received (41 vs. $11 \%, p<0.0001$ ).

Conclusion $\mathrm{MVI}$ is the most frequent poor prognostic marker in our patients with HCC. Local treatment guidelines are effective in yielding comparable outcomes to $B C L C$.
DOI https://doi.org/10.1055/s-0041-1729451 ISSN 2278-330X

How to cite this article: Bhatti H. B. A, Sheikh E. A. A, Mahmud S. U, et al. Clinical Profile and Treatment of Hepatocellular Carcinoma: A Single-Center Experience. South Asian J Cancer 2021;10(2):76-80.
C2021. MedIntel Services Pvt Ltd.

MedIntel Services PvtLtd.. This is an open access article published by Thieme under the terms of the CreativeCommons Attribution-NonDerivative-NonCommercial-License, permitting copying and reproduction so long as the original work is given appropriate credit. Contents may not be used for commercial purposes, or adapted, remixed, transformed or built upon. (https://creativecommons.org/licenses/by-nc-nd/4.0/). Thieme Medical and Scientific Publishers Private Ltd A-12, Second Floor, Sector -2, NOIDA -201301, India 


\section{Introduction}

Cancer remains a leading cause of death worldwide, and hepatocellular carcinoma (HCC) is the third most common cause of cancer death. ${ }^{1}$ The prevalence of HCC continues to rise and the majority of HCC burden lies in Asia and Africa. ${ }^{2}$ In Pakistan, cancer of hepatobiliary origin is considered to be one of the most common cancers in males. The age-standardized rate for HCC in Pakistan is 7.6/100,000/years for males and 2.8/100,000/years for females. ${ }^{3-6}$ Lack of screening, prevalence of risk factors, and limitations in access to treatment result in late presentation and poor survival. ${ }^{7}$ Hepatitis $C$ and hepatitis B continue to be the most common risk factors, while in recent years, a rise in nonviral-related HCC has also been observed. ${ }^{8}$

There are limited published data on HCC in Pakistan and is largely based on hospital registries., ${ }^{3,8}$ It has been shown that only $10 \%$ of patients present in stage- 0 or -A Barcelona clinic liver cancer (BCLC) HCC in Pakistan, and thus, curative treatments can be offered to a selected few. ${ }^{7}$ There are very few tertiary care hospitals where all well-established HCC treatments are offered under one roof. As a result, patients are referred to other local or international centers for treatment. Follow-up of these patients is often difficult, and survival outcomes are rarely known. As a dedicated hepato-pancreato-biliary surgery and liver transplant unit, we are one of the few units in the country to provide all well-established treatments for HCC. With a regular multidisciplinary team (MDT) meeting in place and large flow of HCC patients, we have gathered one of the largest single-center data on HCC patients in Pakistan.

The objective of the current study was to assess the clinical spectrum of HCC in Pakistani patients.

\section{Materials and Methods}

This was a retrospective review of a prospectively maintained database of patients who were referred to the department of hepato-pancreato-biliary surgery and liver transplantation (LT) between 2011 and 2016 with a presumptive diagnosis of HCC. Only patients deemed to have HCC based on the decision of the MDT meeting were included in this study. A total of 1,792 patients with a diagnosis of HCC were seen between December 2011 and August 2018. Patients investigated between December 2011 and September 2016 were included. After the exclusion of patients aged $<18$ years $(n=10)$, a total of 1,490 patients were included in the study.

The diagnosis of HCC was made predominantly on imaging with a dynamic computed tomography scan of the liver and chest. In doubtful cases, magnetic resonance imaging was performed. For the purpose of this study, patient demographics, etiology of liver failure, Child-Turcotte-Pugh (CTP) grade, model for end-stage liver disease (MELD) score, $\alpha$-fetoprotein (AFP) level, and the European Cooperative Oncology Group (ECOG) status were assessed. In addition, tumor size, number, vascular involvement, and the presence of extrahepatic metastasis were also noted. Patients were allocated to different treatment groups based on the BCLC staging algorithm and our local MDT meeting decision. We do not strictly follow BCLC staging for the allocation of patients to various treatments but use our own local guidelines. ${ }^{9}$ The treatment options were grouped as curative (radiofrequency ablation [RFA], percutaneous ethanol injection [PEI], liver resection, LT), palliative (transarterial chemoembolization [TACE], sorafenib), and the best supportive care.

For the assessment of survival, only patients with up to date follow-up were included. Survival was calculated using Kaplan-Meier curves and log-rank test was used to determine significance. We compared survival based on treatment received and MELD score, CTP grade, and AFP level. A value of $p<0.05$ was considered statistically significant. All analyses were performed using the Statistical package for social sciences manufactured by IBM, New York USA. version 20. The hospital ethics committee approved the study.

Table 1 Patient characteristics $(n=1,490)$

\begin{tabular}{|c|c|}
\hline & $n(\%)$ \\
\hline \multicolumn{2}{|l|}{ Gender } \\
\hline Male & $1,099(73.8)$ \\
\hline Female & $391(26.2)$ \\
\hline \multicolumn{2}{|l|}{ Age group (y) } \\
\hline$<40$ & $62(4.1)$ \\
\hline $41-60$ & $865(58.1)$ \\
\hline$>60$ & $563(37.8)$ \\
\hline \multicolumn{2}{|l|}{ Etiology } \\
\hline Hepatitis C & $1,200(80.6)$ \\
\hline Hepatitis B & $133(8.9)$ \\
\hline Hepatitis B and C & $17(1.1)$ \\
\hline $\mathrm{NASH}$ & $39(2.6)$ \\
\hline Others & $101(6.8)$ \\
\hline \multicolumn{2}{|l|}{ MELD score } \\
\hline$<10$ & $803(53.8)$ \\
\hline $11-20$ & 595 (39.9) \\
\hline $21-30$ & $73(4.9)$ \\
\hline $31-40$ & $19(1.2)$ \\
\hline \multicolumn{2}{|l|}{ CTP grade } \\
\hline$A$ & $679(45.5)$ \\
\hline $\mathrm{B}$ & $620(41.6)$ \\
\hline$C$ & $191(12.9)$ \\
\hline \multicolumn{2}{|l|}{ ECOG status } \\
\hline 0 & $76(5.1)$ \\
\hline 1 & $1,212(81.3)$ \\
\hline 2 & $178(11.9)$ \\
\hline 3 & $24(1.6)$ \\
\hline
\end{tabular}

Abbreviations: CTP, Child-Turcotte-Pugh; ECOG, European Cooperative Oncology Group; MELD, model for end-stage liver disease; NASH, Non alcoholic steatohepatitis 
Table 2 Tumor characteristics $(n=1,490)$

\begin{tabular}{|c|c|}
\hline Tumor variables & $n(\%)$ \\
\hline \multicolumn{2}{|l|}{ AFP groups } \\
\hline$\leq 400$ & $831(55.8)$ \\
\hline $400-1,000$ & $69(4.6)$ \\
\hline$>1,000$ & $237(15.9)$ \\
\hline Unknown & $353(23.7)$ \\
\hline \multicolumn{2}{|l|}{ Tumor size $(\mathrm{cm})$} \\
\hline$\leq 2$ & $477(32)$ \\
\hline$>2-5$ & $444(29.8)$ \\
\hline$>5-10$ & $422(28.4)$ \\
\hline$>10$ & $147(9.8)$ \\
\hline \multicolumn{2}{|l|}{$n$} \\
\hline 1 & $724(48.6)$ \\
\hline 2 & $370(24.8)$ \\
\hline 3 & $225(15.1)$ \\
\hline$>3$ & $171(11.5)$ \\
\hline \multicolumn{2}{|l|}{ Lobar involvement } \\
\hline Solitary & $859(57.6)$ \\
\hline Bilobar & $631(42.4)$ \\
\hline \multicolumn{2}{|l|}{ Extra hepatic metastasis } \\
\hline Pulmonary & $33(2.2)$ \\
\hline Lymph nodes & $75(5)$ \\
\hline Adrenal & $4(0.2)$ \\
\hline Peritoneum & $2(0.1)$ \\
\hline Skeletal & $6(0.3)$ \\
\hline Pulmonary + lymph nodes & $4(0.2)$ \\
\hline \multicolumn{2}{|l|}{ Macroscopic vascular involvement } \\
\hline Main or lobar portal vein & $412(27.6)$ \\
\hline Segmental portal vein & $80(5.3)$ \\
\hline
\end{tabular}

Abbreviation: AFP, alpha fetoprotein.

Table 3 Treatment groups based on BCLC and local multidisciplinary team meeting decisions

\begin{tabular}{|l|l|l|}
\hline & $\begin{array}{l}\text { MDT } \\
\text { recommendation } \\
n(\%)\end{array}$ & $\begin{array}{l}\text { BCLC } \\
\text { recommendation } \\
n(\%)\end{array}$ \\
\hline $\begin{array}{l}\text { Liver transplant/ } \\
\text { resection/ } \\
\text { radiofrequency } \\
\text { ablation/percutaneous } \\
\text { ethanol ablation }\end{array}$ & $734(49.3)$ & $543(36.5)$ \\
\hline $\begin{array}{l}\text { Trans arterial } \\
\text { chemoembolization }\end{array}$ & $350(23.5)$ & $205(13.8)$ \\
\hline Sorafenib & $294(19.7)$ & $539(36.1)$ \\
\hline Best supportive care & $112(7.5)$ & $203(13.6)$ \\
\hline
\end{tabular}

Abbreviations: BCLC, Barcelona clinic liver cancer; MDT, multidisciplinary team.

\section{Results}

The mean age was $57.9 \pm 10.1$ years (range: $18-90$ years) The male-to-female ratio was $(1,099 / 391) 2.8: 1$. Hepatitis $\mathrm{B}$ and hepatitis $\mathrm{C}$ were the most common underlying etiological factors in 1,350of 1,490 (90.6\%) patients, as shown in - Table 1. Most patients with HCC had underlying decompensated liver disease (CTP B/C), 811 of 1,490 (54.4\%). However, most of the patients had good functional status (ECOG 0/1), 1,288 of 1,490 (86.4\%).

Median AFP level was $43.6 \quad(0.7-20,000) \mathrm{ng} / \mathrm{mL}$. Extrahepatic metastasis was present in 124 of 1,490 (8.3\%) patients. Macrovascular invasion (MVI) was seen in 492 of 1,490 (33\%) patients, as shown in - Table 2. Out of 492 patients with MVI, 76 (15.4\%) had extrahepatic metastasis. The median MELD score was 10 (5-40). The median CTP score was 7 (5-15). Most frequent sites of extrahepatic metastasis were lymph nodes, 75 of 1,490 (5\%) followed by pulmonary 33 (2.2\%) metastasis.

When compared with BCLC, significantly high numbers of patients were allocated to the LT/RFA/PEI group in the current study. Out of the total, 191 (12.8\%) additional patients were offered potentially curative treatments when compared with BCLC recommendations, as shown in - Table 3. Similarly, TACE was also offered to a significantly high number of patients in the current study. Out of the total, only 304 patients received the recommended treatment and their follow-up was also available. The actuarial 1-year overall survival (OS) with respect to various treatments received was $93,88,65,33$, and $13 \%$, and 5-year OS was $87,64,18,5$, and $0 \%$ as shown in -Fig. 1 .

There was no significant difference in OS based on CTP grade and MELD score in patients with HCC who received various treatments. However, an AFP cut-off of $400 \mathrm{ng} / \mathrm{mL}$ had a significant impact on survival irrespective of treatment received ( 41 vs. $11 \%, p<0.0001$; - Fig. 2 ).

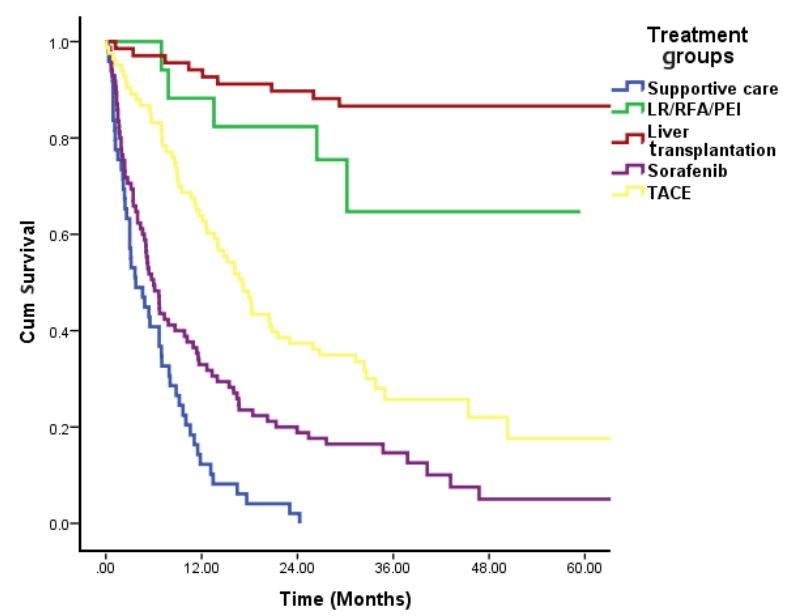

Fig. 1 Actuarial 5-year overall survival with respect to various treatments received. LR, liver resection; PEI, percutaneous ethanol injection; RFA, radiofrequency ablation; TACE, transarterial chemoembolization. 

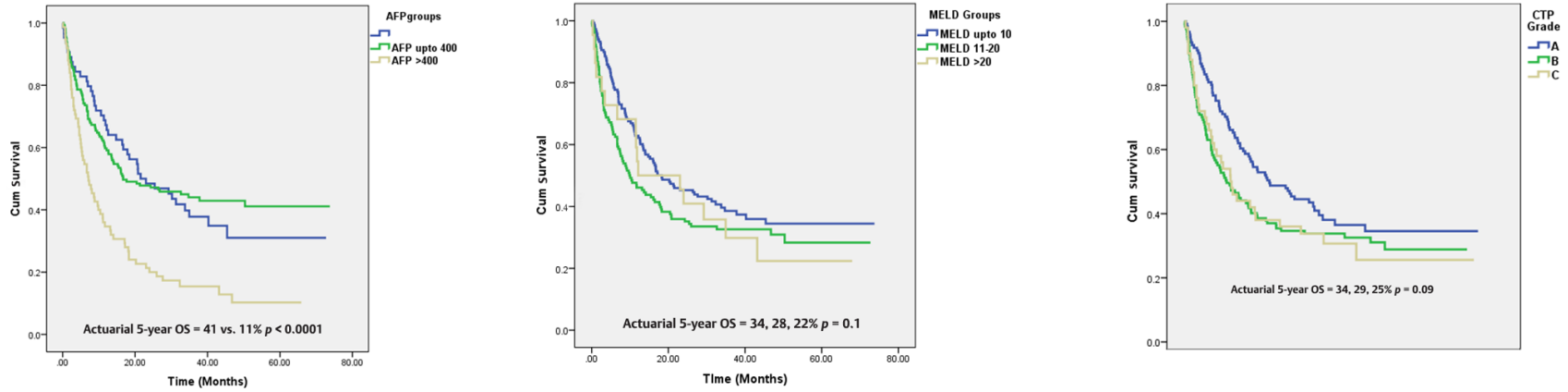

Fig. 2 (A-C) Overall survival with regards to $\alpha$ fetoprotein, Child-Turcotte-Pugh grade and model for end-stage liver disease score. AFP, alpha fetoprotein; CTP, Child-Turcotte-Pugh; MELD, model for end-stage liver disease; OS, overall survival.

\section{Discussion}

The current study reports survival in a relatively large cohort of HCC patients from Pakistan. Pakistani patients with HCC have a similar demographic profile with regard to age group and gender distribution comparable to the rest of the world with certain unique clinical differences.

Macrovascular portal vein involvement was the most frequent aggressive prognostic marker observed in the current study. The rate of malignant portal vein thrombosis very high compared with some of the other regional studies. ${ }^{10,11}$ The current study consisted of all HCC patients seen in the hospital irrespective of whether they received treatment or not. We believe, it demonstrates the HCC stage more reliably than reporting only on patients who received treatment. Many of these patients have good ECOG at presentation. It is obvious that the treatment regimen for this group of patients needs to be individualized and not all patients should be referred for palliation as per BCLC. In the current study, only $15 \%$ of patients with MVI had extrahepatic metastasis. There are emerging data that patients with segmental portal vein involvement can be considered for curative treatments. ${ }^{12-14}$

We observed a marked difference in treatment recommendations from our own local MDT compared with BCLC recommendations. This is primarily due to the restrictive nature of BCLC allocating curative treatments to a small group of HCC patients. ${ }^{15,16}$ In a living donor liver transplantation (LDLT) setting, many centers offer liver transplant to patients with multiple tumors and suboptimal performance status where BCLC recommends palliative treatment. In fact, 191 more patients were eligible for curative treatment compared with BCLC in the current study. A majority of these patients were candidates for LT. A 5-year OS in excess of 80\% clearly demonstrates that patients greatly benefited from LDLT even with tumors outside BCLC or Milan criteria. ${ }^{17,18}$

A unique advantage with LDLT versus deceased donor liver transplantation is the lack of competition for donor organs. LDLT implies purely an altruistic donation to a sick patient from a member of his family. Thus, transplant criteria can be carefully extended. We have shared our algorithm for allocating treatment to HCC patients elsewhere. ${ }^{9}$
AFP is a well-established prognostic marker in HCC patients. Several patients with HCC present with normal AFP levels despite underlying HCC. The current study showed that irrespective of treatment received, patients with low AFP $(<400 \mathrm{ng} / \mathrm{dL}$ ) had a significantly better OS. There is a case to be more aggressive in patients with low AFP, particularly those with segmental portal vein involvement expecting improved outcomes in these patients. ${ }^{19-21}$ However, there remains an element of selection bias as patients who were offered transplant were less likely to have very high AFP levels even in the presence of HCC outside Milan criteria. No significant difference in outcomes was seen with increasing MELD score and child class. This supports the role of aggressive treatments like transplantation in patients with advanced liver failure and underlying HCC. In fact, liver transplant is an excellent treatment option in these patients, provided the HCC falls within acceptable transplant criteria.

Among palliative treatments, TACE was offered to more patients when compared with BCLC. This is due to our policy of offering TACE to patients with segmental or branch portal vein tumor thrombus and/or limited extrahepatic disease. ${ }^{22,23} \mathrm{~A} 5$-year survival close to $20 \%$ is again reflective of an expanding role and indications of TACE in patients managed with palliation.

\section{Conclusion}

The current study reports on a large cohort of patients with HCC, seen at a single center in Pakistan. It shows that with careful patient selection, a good number of HCC patients can be offered curative treatments. LT has a promising role in management of liver cirrhosis since it treats both the HCC and liver failure. There is a case to be more aggressive in patients which vascular invasion and no extrahepatic metastasis since this represents a large group of our patients. However, the appropriate group with expected favorable outcomes remains to be defined. There is a need to improve screening methods in patients with risk factors for HCC to aid early diagnosis and treatment.

Financial Support and Sponsorship

Nil. 
Conflicts of interests.

There are no conflicts of interest to declare.

\section{References}

1 Bruix J, Reig M, Sherman M. Evidence-based diagnosis, staging, and treatment of patients with hepatocellular carcinoma. Gastroenterology 2016;150(4):835-853

2 Jemal A, Center MM, DeSantis C, Ward EM. Global patterns of cancer incidence and mortality rates and trends. Cancer Epidemiol Biomarkers Prev 2010;19(8):1893-1907

3 Badar F, Mahmood S. Hospital-based cancer profile at the Shaukat Khanum Memorial Cancer Hospital and Research Centre, Lahore, Pakistan. J Coll Physicians Surg Pak 2015;25(4):259-263

4 Bhurgri Y, Bhurgri A, Hassan SH, et al. Cancer incidence in Karachi, Pakistan: first results from Karachi Cancer Registry. Int J Cancer 2000;85(3):325-329

5 Bhurgri Y, Bhurgri A, Pervez S, et al. Cancer profile of Hyderabad, Pakistan 1998-2002. Asian Pac J Cancer Prev 2005; 6(4):474-480

6 Bhurgri Y, Pervez S, Kayani N, et al. Cancer profile of Larkana, Pakistan (2000-2002) Asian Pac J Cancer Prev 2006; 7(4):518-521

7 Hafeez Bhatti AB, Dar FS, Waheed A, Shafique K, Sultan F, Shah NH. Hepatocellular carcinoma in Pakistan: National trends and global perspective. Gastroenterol Res Pract 2016; 2016:5942306

8 Butt AS, Hamid S, Wadalawala AA, et al. Hepatocellular carcinoma in Native South Asian Pakistani population; trends, clinico-pathological characteristics \& differences in viral marker negative \& viral-hepatocellular carcinoma. BMC Res Notes 2013;6:137

9 Shah NH, Dar FS, Bhatti AB, Rana A, Salih M. Assigning treatment to HCC patients for transplantation: utility of a new decision-making tool. Ann Transplant 2016;21:668-674

10 Aljumah AA, Kuriry H, AlZunaitan $\mathrm{M}$, et al. Clinical presentation, risk factors, and treatment modalities of hepatocellular carcinoma: a single tertiary care center experience. Gastroenterol Res Pract 2016;2016:1989045

11 Alswat KA, Sanai FM, Altuwaijri M, et al. Clinical characteristics of patients with hepatocellular carcinoma in a middle eastern population. Hepat Mon 2013;13(5):e7612

12 Han DH, Joo DJ, Kim MS, et al. Living donor liver transplantation for advanced hepatocellular carcinoma with portal vein tumor thrombosis after concurrent chemoradiation therapy. Yonsei Med J 2016;57(5):1276-1281

13 Chong JU, Choi GH, Han DH, et al. Downstaging with localized concurrent chemoradiotherapy can identify optimal surgical candidates in hepatocellular carcinoma with portal vein tumor thrombus. Ann Surg Oncol 2018;25(11):3308-3315

14 Choi HJ, Kim DG, Na GH, et al. The clinical outcomes of patients with portal vein tumor thrombi after living donor liver transplantation. Liver Transpl 2017;23(8):1023-1031

15 Barman PM, Su GL. Limitations of the barcelona clinic liver cancer staging system with a focus on transarterial chemoembolization as a key modality for treatment of hepatocellular carcinoma. Clin Liver Dis (Hoboken) 2016;7(2):32-35

16 Vitale A, Peck-Radosavljevic M, Giannini EG, et al. Personalized treatment of patients with very early hepatocellular carcinoma. J Hepatol 2017;66(2):412-423

17 Bhatti ABH, Dar FS, Qureshi AI, et al. Living donor liver transplantation for hepatocellular carcinoma: a single-center experience from Pakistan. J Clin Exp Hepatol 2019;9(6):704-709

18 Mazzaferro V, Regalia E, Doci R, et al. Liver transplantation for the treatment of small hepatocellular carcinomas in patients with cirrhosis. N Engl J Med 1996;334(11):693-699

19 Cerban R, Ester C, Iacob S, et al. Predictive factors of tumor recurrence and survival in patients with hepatocellular carcinoma treated with transarterial chemoembolization. J Gastrointestin Liver Dis 2018;27(4):409-417

20 Toro A, Ardiri A, Mannino M, et al. Effect of pre- and post-treatment $\alpha$-fetoprotein levels and tumor size on survival of patients with hepatocellular carcinoma treated by resection, transarterial chemoembolization or radiofrequency ablation: a retrospective study. BMC Surg 2014;14:40

21 Lai Q Iesari S, Melandro F, Mennini G, Rossi M, Lerut J. The growing impact of alpha-fetoprotein in the field of liver transplantation for hepatocellular cancer: time for a revolution. Transl Gastroenterol Hepatol 2017;2:72

22 Malagari K, Moschouris H, Kiakidis T, et al. Five-years outcome analysis of 142 consecutive hepatocellular carcinoma patients treated with doxorubicin eluting microspheres 30-60 $\mu \mathrm{m}$ : results from a single-centre prospective phase II trial. Cardiovasc Intervent Radiol 2019;42(11):1551-1562

23 Kirstein MM, Voigtländer T, Schweitzer N, et al. Transarterial chemoembolization versus sorafenib in patients with hepatocellular carcinoma and extrahepatic disease. United European Gastroenterol J 2018;6(2):238-246 